\title{
Room temperature photoluminescence from nanostructured amorphous carbon
}

\author{
S. J. Henley, ${ }^{\text {a) }}$ J. D. Carey, and S. R. P. Silva \\ Nano Electronics Centre, Advanced Technology Institute, School of Electronics and Physical Sciences, \\ University of Surrey, Guildford GU2 7XH, United Kingdom
}

(Received 11 August 2004; accepted 25 October 2004)

\begin{abstract}
Visible room-temperature photoluminescence (PL) was observed from hydrogen-free nanostructured amorphous carbon films deposited by pulsed laser ablation in different background pressures of $\operatorname{argon}\left(P_{\mathrm{Ar}}\right)$. By varying $P_{\mathrm{Ar}}$ from 5 to $340 \mathrm{mTorr}$, the film morphology changed from smooth to rough and at the highest pressures, low-density filamentary growth was observed. Over the same pressure regime an increase in the ordering of $s p^{2}$ bonded $\mathrm{C}$ content was observed using visible Raman spectroscopy. The origin of the PL is discussed in terms of improved carrier localization within an increased $s p^{2}$ rich phase. (C) 2004 American Institute of Physics. [DOI: 10.1063/1.1839641]
\end{abstract}

The large variation in the properties of carbon films, deposited by different techniques, principally arises from the different bonding configurations available. Carbon can hybridize with varying percentages of $s p^{1}$ (acetylene-like), $s p^{2}$ (graphite-like), and $s p^{3}$ (diamond-like) bonding. ${ }^{1}$ The pulsed laser ablation (PLA) of graphite, in vacuum, has typically been used to produce smooth, hard, high density amorphous carbon (a-C) films with high $s p^{3}$ content (up to 90\%) ) $^{2,3}$ due to the local densification, on the impact of high energy $\mathrm{C}$ atoms/ions, favoring the formation of the $s p^{3}$ phase. The optical properties of carbon films are largely governed by the $s p^{2}$ phase, with the tribological properties determined by the $s p^{3}$ phase. However, in these nanocomposite materials, some care must be exercised in extracting and interpretation of the Tauc band gap and the Urbach energy.

Photoluminescence (PL) in amorphous carbon, is thought to occur due to the radiative recombination of electrons and holes in the band-tail states created by $s p^{2}$ rich clusters. ${ }^{4,5} \mathrm{PL}$ has been extensively studied for polymeric a-C:H films, ${ }^{4,6,7}$ where an increase in the PL intensity has been observed for increasing $\mathrm{H}$ content. ${ }^{7}$ In this letter we investigate the PL properties of hydrogen-free a-C films deposited by PLA of graphite in background pressures of argon. Unlike in vacuum, these conditions have been shown to produce low density nanostructured amorphous carbon (NAC) films. ${ }^{8,9}$ Such films have been shown to have useful properties including low thresholds for electron emission ${ }^{10}$ and high porosities. ${ }^{9}$

Films were deposited by PLA of a $99.999 \%$ pure pyrolytic graphite target (Kurt J. Lesker) using a Lambda-Physik LPX 210i excimer laser operating at $248 \mathrm{~nm}$. The laser pulse was focused onto the rotating target, producing a fluence of $\sim 6 \mathrm{~J} / \mathrm{cm}^{2}$. The growth chamber was evacuated using a turbomolecular pump to $9 \times 10^{-8}$ Torr, then backfilled with argon to the required pressure. Films were deposited onto $\mathrm{Si}$ substrates at a range of different background pressures of $\operatorname{argon}\left(P_{\mathrm{Ar}}\right)$ from 5 to 340 mTorr. The target to substrate distance was fixed at $6 \mathrm{~cm}$. Between 2000 and 5000 laser shots were used, depending on the background pressure in order to keep the film thickness constant as it was affected by $P_{\text {Ar }}$.

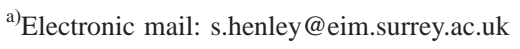

The morphology of the films was investigated in a Hitachi S4000 field emission gun scanning electron microscope (SEM). The structure and bonding configuration in the films was investigated by visible Raman spectroscopy (Renishaw Ramanscope) using two different laser wavelengths 488 and $514 \mathrm{~nm}$. The PL measurements were also performed using the system with the $488 \mathrm{~nm}$ laser, which was also equipped with a cryostat (Oxford Instruments) that allowed measurements at substrate temperatures down to $6 \mathrm{~K}$.

After deposition the surface morphology of the films was examined in the SEM. Figure 1 shows SEM images of a range of samples deposited at different $P_{\mathrm{Ar}}$. At the lowest $P_{\mathrm{Ar}}$ used ( 5 mTorr) the films deposited were hard, scratch resistant and demonstrated a smooth morphology [see Fig. 1(a)]. As $P_{\text {Ar }}$ was increased to 40 mTorr [Fig. 1(b)] the films became much rougher and nanoscale clustering was observed in the SEM. The size of these clusters is estimated as $15 \pm 3 \mathrm{~nm}$. As $P_{\text {Ar }}$ was again increased to $100 \mathrm{mTorr}$, theclustering of the film become more pronounced and the clusters became larger in size, $\sim 45 \mathrm{~nm}$. The films deposited at 100 mTorr could be easily scratched off with a pinpoint, indicating a much lower hardness. By 340 mTorr the film morphology had changed from nanoscale clusters to more

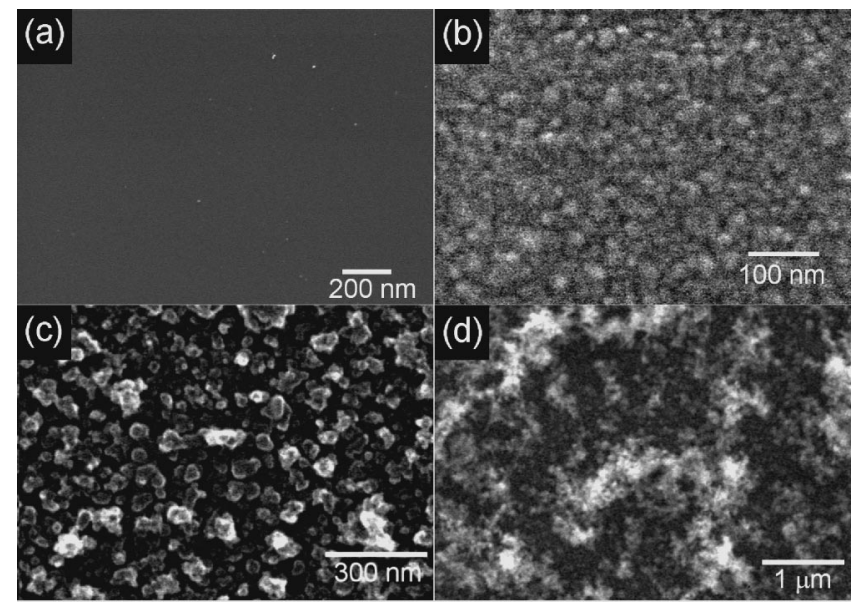

FIG. 1. SEM images of a range of samples deposited at different $P_{\mathrm{Ar}}$ : (a) 5, (b) 40, (c) 100, and (d) 340 mTorr. Note the different scale bars on the images. 


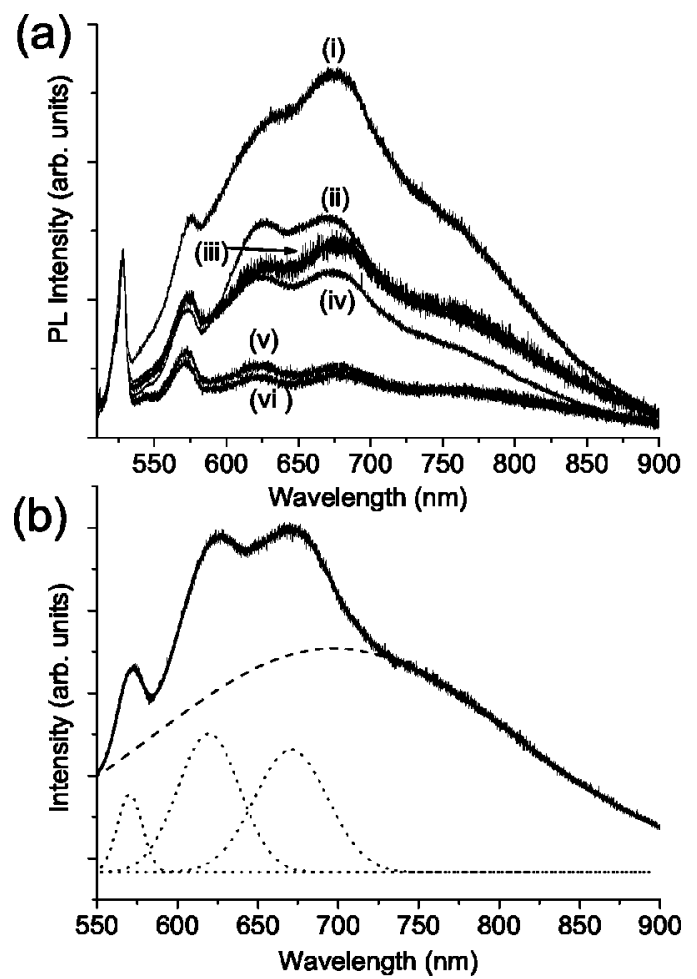

FIG. 2. Plots of PL spectra using $488 \mathrm{~nm}$ excitation: (a) spectra for a range of samples deposited at different $P_{\text {Ar. }}$. Trace (i) corresponds the film grown with $P_{\mathrm{Ar}}=150$, (ii) 80 , (iii) 50, (iv) 40, (v) 15 , and (vi) $11 \mathrm{mTorr}$; (b) plot of the four Gaussian fits to spectrum (ii).

filamentary growth. It was possible to remove these films by rubbing the surface, indicating a further reduction in film density with increasing $P_{\mathrm{Ar}}$.

After characterizing the structure of the films, the optical properties were investigated using Raman and PL spectroscopy. Figure 2(a) shows a series of PL spectra taken at room temperature using visible $(488 \mathrm{~nm})$ excitation. These spectra show a variety of features. First we observe the sharp Raman signal at around $529 \mathrm{~nm}$. For comparison these spectra have all been normalized to the intensity of the Raman peak. As the thickness and porosity of the films varied with the background pressure, normalizing to the Raman peak is the only sensible method for comparing the luminescence. To a fair approximation, the Raman signal will be proportional to the film thickness. Higher in wavelength than the Raman signal is a broad PL feature that appears to be split into different bands. Figure 1(b) shows the spectra obtained from the film deposited at $P_{\mathrm{Ar}}=80 \mathrm{mTorr}$, and has been fitted with four Gaussian functions to separate the contribution of the different bands. The first band, at around $575 \mathrm{~nm}$, is quite sharp and appears not to vary significantly in intensity with $P_{\mathrm{Ar}}$, so maybe the second order Raman peak. The other bands however, swap in relative intensity at different values of $P_{\mathrm{Ar}}$. Similar shaped PL spectra have been observed from a-C:H samples deposited by dc-magnetron sputtering. ${ }^{7}$ The majority of the PL intensity detected appears to come from the broad band centered at around $700 \mathrm{~nm}$. The intensity of this band increased as the $P_{\mathrm{Ar}}$ was raised. PL measurements were also performed at low substrate temperatures $(7 \mathrm{~K})$, again using $488 \mathrm{~nm}$ excitation, but only a weak temperature dependence of the PL was observed. A similar weak temperature dependence of the PL intensity has been observed in a-C:H samples ${ }^{11}$ and can be associated with the broad band tail

Downloaded 17 Dec 2004 to 131.227.4.58. Redistribution subject

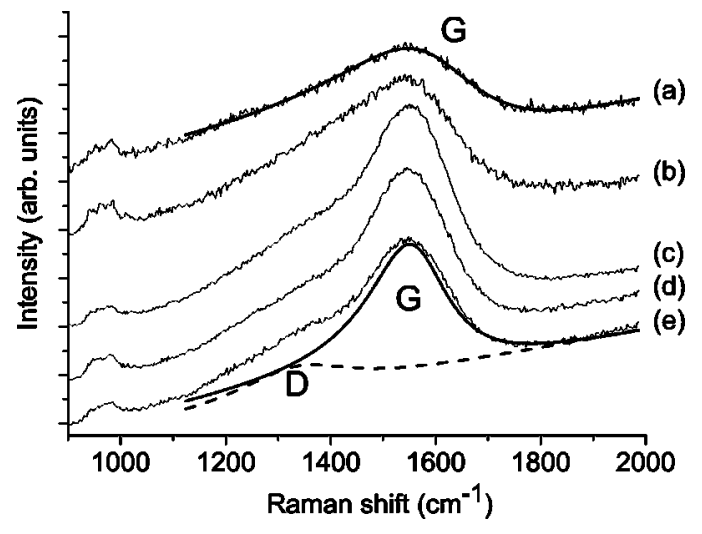

FIG. 3. Plots of visible Raman spectra for NAC films. Trace (a) corresponds to the film grown with $P_{\mathrm{Ar}}=5$, (b) 11 , (c) 50, (d) 150, and (e) $340 \mathrm{mTorr}$. Fits to the data are included for traces (a) only using the $G$ peak and (e) using both $D$ and $G$ peaks.

states which give rise to highly localized and polarized intra$s p^{2}$-cluster PL emission.

The Raman spectrum for each sample was measured using $514 \mathrm{~nm}$ excitation. A selection of these spectra are shown in Figs. 3(a) and 3(b). The Raman spectra are characterized as a combination of two bands, typically labeled the $D$ and $G$ peaks and are attributed to the breathing mode of aromatic rings and in-plane bondstretching of pairs of $s p^{2}$ bonded $\mathrm{C}$ atoms, respectively. ${ }^{12-14}$ For $514 \mathrm{~nm}$ excitation the $D$ peak is typically located at $1335 \mathrm{~cm}^{-1}$ and the $G$ peak is typically found in the range $1580 \pm 20 \mathrm{~cm}^{-1}$. In order to characterize the films, the Raman spectra were fitted using a BreitWigner-Fano line for the $G$ peak and a Lorentzian for the $D$ peak. ${ }^{13}$ For the films deposited at low $P_{\text {Ar }}$ (below $\sim 15$ mTorr) the complete Raman line shape can be fitted without the need for the $D$ peak. This is characteristic of ta-C, where the ratio of the peak intensities of the $D$ and $G$ peaks $\left(I_{D} / I_{G}\right)$ tends towards zero as the fraction of $s p^{3}$ bonded $\mathrm{C}$ increases. As $P_{\mathrm{Ar}}$ increased the Raman line shapes could only be fitted by including an increasing contribution of the $D$ peak. This indicated that the fraction of $s p^{2}$ bonded $\mathrm{C}$ present in the films increased, accompanied by an increase in ordering due to the formation of larger clusters of aromatic rings. ${ }^{13}$ The Raman data presented here are similar to those of Siegal et al. ${ }^{9}$ who saw little change in the Raman spectra with increasing $P_{\mathrm{Ar}}$, during PLA, in the range 175-300 mTorr. We also observe that, although the density of the films is very low, the Raman spectra are similar to those of high density a-C, unlike the films deposited by cluster-assembly. ${ }^{15}$

For comparison the ratio $I_{D} / I_{G}$ is plotted as a function of $P_{\mathrm{Ar}}$ in Fig. 4(a). Also plotted [Fig. 4(b)] is the relative PL intensity over the same pressure range. This was estimated by taking the area of the large broad band observed during the fitting of the PL spectra [see Fig. 2(b)]. It can be readily observed that the increase in the PL intensity correlates well with the increase in $I_{D} / I_{G}$. This suggests that the enhanced PL, with increased $P_{\mathrm{Ar}}$, is a consequence of carrier localization within an increasing number of $s p^{2}$ rich clusters.

As $P_{\mathrm{Ar}}$ is increased the velocity of carbon atoms and ions in the plume is expected to decrease due to collisions. By imaging the optical emission from the plume (Henley et al., unpublished), it was demonstrated that the $\mathrm{C}^{+}$ion velocity reduced from $\sim 42 \mathrm{~km} / \mathrm{s}$ in vacuum (equivalent to a kiAlP license or copyright, see http://apl.aip.org/apl/copyright.jsp 


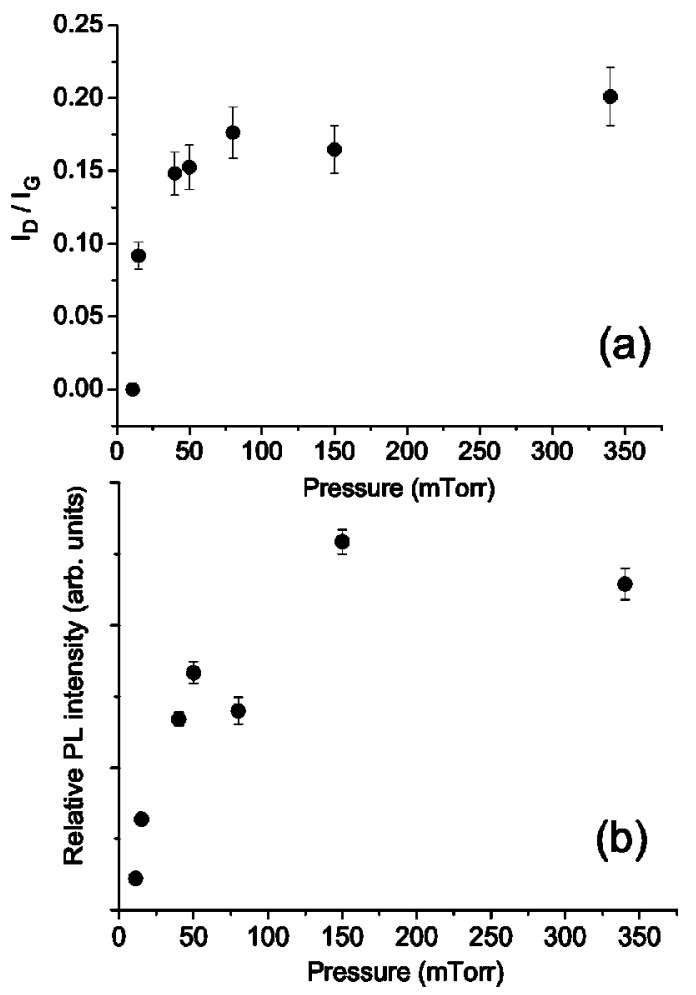

FIG. 4. (a) Plot of $I_{D} / I_{G}$ obtained from fits to the Raman data shown in Fig. 3; (b) plot of the relative PL intensity as a function of $P_{\mathrm{Ar}}$.

netic energy of $\sim 100 \mathrm{eV}$, a regime in which dense ta-C films are deposited), to diffusion limited velocities at 340 mTorr. At the higher $P_{\mathrm{Ar}}$ the formation of carbon clusters in the gas phase, along with the reduction in the $\mathrm{C}$ atom/ion velocity by impacts is expected to produce low density $s p^{2}$ rich films, as is observed. The coalescence of these clusters produces the rough nanostructure observed in the SEM. However, by $P_{\text {Ar }}=340$ mTorr, the observed filamentary nature of the films may be a due to the clusters arriving at energies too low for coalescence to occur.

In conclusion, it has been shown that the PL intensity from hydrogen-free a-C, grown by PLA, increases as the $P_{\mathrm{Ar}}$ used during deposition, was increased. As the background pressure was raised the film morphology changed from smooth coatings to rough, nanostructured films and then onto low-density filamentary growth. This transition was accompanied by an increase in the ordering of the $s p^{2}$ content of the films, as measured by Raman spectroscopy. It is suggested that an increased $P_{\mathrm{Ar}}$, during deposition, increases the density of $s p^{2}$ rich clusters in the film. Carrier localization within these clusters is then responsible for the enhanced PL.

S.J.H. would like to thank J. Filik, G. Fuge, P.W. May, and H.M.B. Darwish from the University of Bristol for assistance in obtaining the Raman and PL data. The authors would also like to thank the EPSRC Portfolio Grant and the CBE Programme for funding the project.

${ }^{1}$ S. R. P. Silva, J. D. Carey, R. U. A. Khan, E. G. Gernster, and J. V. Anguita, in Handbook of Thin Film Materials, edited by H. S. Nalwa (Academic, New York, 2002), and references therein.

${ }^{2}$ A. A. Voevodin and M. S. Donley, Surf. Coat. Technol. 82, 199 (1996).

${ }^{3}$ M. P. Siegal, J. C. Barbour, P. N. Provencio, D. R. Tallant, and T. A. Friedmann, Appl. Phys. Lett. 73, 759 (1998).

${ }^{4}$ F. DeMichelis, S. Schreiter, and A. Tagliaferro, Phys. Rev. B 51, 2143 (1995).

${ }^{5}$ S. R. P. Silva, Rusli, J. Schwan, G. A. J. Amaratunga, and J. Robertson, Philos. Mag. B 74, 369 (1996).

${ }^{6}$ J. Robertson, Phys. Rev. B 53, 16302 (1996)

${ }^{7}$ B. Marchon, J. Gui, K. Grannen, G. C. Rauch, J. Ager, S. R. P. Silva, and J. Robertson, IEEE Trans. Magn. 33, 3148 (1997).

${ }^{8}$ Z. Geretovszky, T. Haraszti, T. Szörényi, F. Antoni, and E. Fogarassy, Appl. Surf. Sci. 208-209, 566 (2003).

${ }^{9}$ M. P. Siegal, D. L. Overmyer, R. J. Kottenstette, D. R. Tallant, and W. G. Yelton, Appl. Phys. Lett. 80, 3940 (2002).

${ }^{10}$ B. S. Satyanarayana, J. Robertson, and W. I. Milne, J. Appl. Phys. 87, 3126 (2000).

${ }^{11}$ M. Liao, Z. Feng, S. Yang, C. Chai, Z. Liu, J. Yang, and Z. Wang, Solid State Commun. 121, 287 (2002).

${ }^{12}$ M. A. Tamor and W. C. Vassel, J. Appl. Phys. 76, 3823 (1994).

${ }^{13}$ A. C. Ferrari and J. Robertson, Phys. Rev. B 61, 14095 (2000).

${ }^{14}$ J. Schwan, S. Ulrich, V. Batori, H. Ehrhardt, and S. R. P. Silva, J. Appl. Phys. 80, 440 (1996).

${ }^{15}$ E. Barborini, P. Piseri, A. L. Bassi, A. C. Ferrari, C. E. Bottani, and P. Milani, Chem. Phys. Lett. 300, 633 (1999). 